\title{
Endogenous capacities and price competition: The role of demand uncertainty
}

\author{
María-Ángeles de Frutos, Natalia Fabra* \\ Universidad Carlos III de Madrid, Spain
}

\section{A R T I C L E I N F O}

\section{JEL classification:}

D43

D80

L11

Keywords:

Investment

Price competition

Demand uncertainty

Submodular games

Cournot game

\begin{abstract}
A B S T R A C T
We analyze the role of demand uncertainty in markets of fixed size, in which firms take long run capacity decisions prior to competing in prices. We characterize the set of subgame perfect Nash equilibria under various assumptions regarding the nature and timing of demand uncertainty. In order to prove equilibrium existence, we identify a sufficient condition for the capacity choice game to be submodular. This condition resembles the standard downward sloping marginal revenue condition used in Cournot games. A robust conclusion of the analysis is that equilibrium capacity choices are asymmetric, even when firms are ex ante identical. Concerning the equivalence between the capacity price game and the Cournot game, we find that with inelastic demands, the equilibria of the former belong to the equilibrium set of the latter. However, as compared to the Cournot game, the capacity price game leads to lower prices and generates price dispersion.
\end{abstract}

\section{Introduction}

We analyze a two stage game in which firms take capacity decisions under demand uncertainty, and then engage in price competition. We focus on markets with price inelastic demands, also referred to as "markets of fixed size" (Cripps and Ireland, 1988). This specification is well suited to analyze the performance of several industries in which long run investments are followed by price competition. This is for instance the case of electricity markets, in which generators invest in long lived assets in order to meet fluctuating demands. Given that the vast majority of consumers pay fixed tariffs irrespective of market based prices, their demands tend to be quite inelastic, at least in the short run (Fabra et al., 2009). Large scale communication networks, particularly the Internet, share similar features in that service providers first invest in capacities under uncertain market conditions, and subsequently engage in price competition to attract the demand "flows" of internet services (Acemoglu et al., 2009).

Several papers have tried to understand the role of demand uncertainty in capacity price games. The interest on this issue dates back to Kreps and Scheinkman's (1983) seminal paper, in which they conjectured that "noise" in the demand function would destroy their

* Corresponding author. Department of Economics, Calle Madrid 126, 28903 Getafe, Madrid, Spain.

E-mail addresses: frutos@eco.uc3m.es (M.-Á. de Frutos), natalia.fabra@uc3m.es (N. Fabra). main result, namely, that capacity precommitment followed by price competition yields Cournot outcomes. Gal Or (1984) provided the first analysis on this topic in a model with inelastic demand, but limited to the case in which firms take capacity and price decisions prior to observing the actual demand realization. ${ }^{1}$ Since then, other papers have analyzed the same topic under the assumption of downward sloping demand; for instance, Reynolds and Wilson (2000) and Lepore (2008) focus on the case in which demand is realized before firms engage in price competition, whereas Hviid (1990) focuses on the case in which demand is realized ex post. ${ }^{2}$ Broadly speaking, all these papers arrive at two main conclusions: first, the equivalence between Bertrand and Cournot outcomes remains true under some, but not all model specifications; and second, the capacity game may fail to have a symmetric pure strategy equilibrium. ${ }^{3}$ Our model contributes to this literature by considering

\footnotetext{
${ }^{1}$ Cripps and Ireland (1988) analyze a similar model as Gal-Or (1984)'s, but focus on the game with demand certainty.

2 Gabszewicz and Poddar (1997) and Grimm and Zoettl (2006) also consider future demand uncertainty at the investment stage, but assume that firms subsequently compete by choosing quantities rather than prices. The Cournot assumption has important implications on investment incentives and equilibrium outcomes. In particular, the existence of a symmetric equilibrium in capacity choices is guaranteed.

${ }^{3}$ An exception is Gal-Or (1984), who predicted symmetric capacity choices. Unfortunately, her analysis contained a mistake which has not yet been solved, despite being well-known: she only analyzed the pricing subgame starting at symmetric capacity choices and was therefore unable to correctly assess the effects of deviations from the symmetric capacity pairs on second stage expected profits.
} 
the case of price inelastic demands for a wide family of demand distribution functions and various formulations on the nature and timing of demand uncertainty. More specifically, we allow demand to follow a continuous or a two point distribution, and we analyze the cases in which demand is realized either before or after firms take their pricing decisions.

We characterize the subgame perfect Nash equilibria of the two stage game. We confirm that the capacity choice game with inelastic demand and ex ante identical firms does not have symmetric pure strategy equilibria; indeed, it only has two asymmetric outcome equivalent pure strategy equilibria. The reason why market outcomes are asymmetric is that large and small firms face asymmetric marginal returns to investment. In particular, one firm prefers to be small in order to induce less aggressive pricing by its rival, thus fostering an increase in its expected profits despite losing output in states at which the firm sells at capacity. Mathematically, this gives rise to kinked profit functions and discontinuous best response correspondences which never cross the 45 degree line. Proving existence of pure strategy equilibria in this set up is a challenging problem. However, we identify a sufficient condition on the demand distribution function that allows to apply the theory of submodular games (Topkis, 1979). The economic interpretation of this sufficient condition is analogous to the standard downward sloping marginal revenue condition used in Cournot games. Since this condition does not depend on capacity cost structures, the capacity game remains submodular under general capacity cost specifications, including nonlinear and/or asymmetric cost functions across firms. For this reason, the basic model can be easily extended to show that ex ante asymmetries in capacity costs enhance ex post asymmetries. In sum, we provide further support to the claim that asymmetric market structures are a robust prediction in capacity choice models. ${ }^{4}$

Endogenous asymmetries also arise in a broad family of studies in applied microeconomics. They all share two important features with our model: some form of non concavity in payoffs, and submodularity in first period actions (Amir et al., 2010). Among these studies, our approach for proving equilibrium existence closely follows Amir and Wooders (2000), who analyze a two stage game of R\&D investments followed by product market competition.

Besides from generating asymmetries, demand uncertainty also has a crucial effect on equilibrium multiplicity. Whereas the certain demand game generates a continuum of equilibria, including a symmetric one, the game with continuous demand uncertainty picks just two of them. ${ }^{5}$ If demand follows a two point distribution, there are more equilibria that survive the introduction of demand uncertainty; notably, symmetric and asymmetric equilibria may coexist. However, if none of the two possible states is sufficiently likely, only the asymmetric equilibria survive.

We also find that the nature of equilibrium capacity choices are not affected by the timing of demand uncertainty, i.e., by whether demand is realized either before or after pricing decisions. More specifically, equilibrium capacities are asymmetric in both games because both depict asymmetries in firms' marginal returns to investment. Furthermore, aggregate capacity is also unchanged, as in both games the marginal capacity unit is sold at the reservation

\footnotetext{
${ }^{4}$ See Reynolds and Wilson (2000) for a survey on capacity choice models that also give rise to asymmetric outcomes.

${ }^{5}$ In this respect, our model is also related to Klemperer and Meyer's (1989) seminal paper in which firms facing uncertain demand compete by choosing supply functions. They find that the introduction of demand uncertainty dramatically shrinks the set of equilibria, and conclude that the equilibrium is unique only if the support of the demand distribution function is unbounded. Even though our approach substantially differs from theirs, it is not surprising that our conclusions concerning the effect of demand uncertainty are similar. In both scenarios, continuous demand uncertainty reduces multiplicity of equilibria since it forces each firm's strategic decision to be optimal against a range of possible demand functions.
}

price. Nevertheless, equilibrium outcomes (i.e., individual firms' capacities and expected prices) may differ.

Last, our results serve to partially vindicate Kreps and Scheinkman's. Namely, with inelastic and uncertain demand, capacity precommitment followed by price competition yields Cournot outcomes in the following sense: the set of equilibrium capacity choices of the two stage game is contained in the set of Cournot equilibria. However, whereas both games yield the same level of aggregate capacity (and are thus equivalent in terms of welfare), the two stage game leads to lower prices and generates price dispersion, as correctly pointed out by Gal Or (1984).

The structure of the paper is as follows. Section 2 describes and solves the basic model, with and without demand uncertainty. In Section 3, we vary and extend the basic model in several directions: general cost functions and ex ante asymmetries, two point demand distributions, and demand uncertainty at the pricing stage. Section 4 compares the results obtained in the basic model with those of its certainty equivalent game and the Cournot game. The last section concludes and proofs are included in Appendix A.

\section{The model}

Two symmetric firms, $i=1,2$, supply a homogeneous good to a mass $\theta$ of infinitesimal and identical buyers. Each buyer consumes exactly one unit of the good if its market price is less than the reservation price, normalized to unity, and if it is available on the market. Hence, total demand is $\theta$ as long as the market price is not greater than 1 . The number of buyers $\theta$ is randomly determined in the unit interval $[0,1]$ according to the continuous cumulative distribution function $G(\theta)$, which satisfies the following properties:

Assumption 1. $G$ has a continuously differentiable density $g$, bounded away from zero in the interior of its support.

We consider a two stage non cooperative game. In the first stage, firms simultaneously choose their capacities $k_{i}, i=1,2$, at unit cost $c \in(0,1)$. Capacity decisions are irreversible and become publicly known. We let $k=\min \left\{k_{1}, k_{2}\right\} \leq k^{+}=\max \left\{k_{1}, k_{2}\right\}$, and refer to the firm with capacity $k^{-}$as "the small firm" and to the firm with capacity $k^{+}$as "the large firm"; aggregate capacity $k^{-}+k^{+}$is denoted by $K$. Next, the number of buyers $\theta$ is realized and observed by all firms. In the second stage, firms simultaneously choose prices. Consumers buy first from the firm with the low price until its capacity is exhausted, and the residual buyers are served by the high priced firm. If firms' prices are equal, consumers split equally between the two. ${ }^{6}$ Finally, each firm sells its production at its own price. Production entails zero marginal costs up to each firm's capacity, while producing above capacity is infinitely costly. Firms are assumed to be risk neutral and to maximize expected profits.

We search for the subgame perfect equilibria of the two stage game. We allow firms to play mixed strategies at the pricing subgame, but focus on pure strategies at the investment stage (mixed strategies in capacity choices are difficult to either justify or interpret). Accordingly, each firm's strategy is a pair specifying its capacity choice, and its distribution function over prices given both firms' capacities. We solve the game by backwards induction.

\footnotetext{
${ }^{6}$ As noted by Cripps and Ireland (1988), the homogeneous nature of the market means that if buyers are rationed at a firm, the sets of successful and unsuccessful buyers are identical. Hence, our results are valid regardless of the rationing rule, including those investigated by Davidson and Deneckere (1986), Osborne and Pitchik (1986) or Lepore (2008), among others. Our results are also independent of the tiebreaking rule.
} 


\subsection{Pricing stage}

Consider any subgame starting after demand has been realized, and firms have observed each other's capacity choices. Equilibrium pricing in this case was first analyzed by Cripps and Ireland (1988), ${ }^{7}$ whose results we report next (see also Osborne and Pitchik, 1986):

Proposition 1. (Cripps and Ireland (1988)) In the pricing stage, for given $\theta$ and $k^{-} \leq k^{+}$, there is a unique equilibrium which satisfies the following:

(i) (Region I) If $\theta \leq k^{-}$, there exists a unique pure strategy equilib rium in which both firms set prices equal to (zero) marginal cost and make zero expected profits.

(ii) (Region II) If $k^{-}<\theta<K$, a pure strategy equilibrium fails to exist. In the unique mixed strategy equilibrium, the large firm makes expected profits $\left[\theta-k^{-}\right]$, whereas the small firm makes a fraction $\frac{k}{\min \left\{\theta, k^{+}\right\}}$of the large firm's expected profits. ${ }^{8}$

(iii) (Region III) If $\theta \geq K$, in the unique equilibrium both firms set prices equal to consumers' reservation price (which equals 1 ), and they both sell at capacity.

Equilibrium pricing behavior depends on the relationship between demand and capacities. For demand realizations in Region I, since either firm has enough capacity to serve total demand, competition drives prices down to marginal cost, and firms make zero expected profits. For demand realizations in Region III, since there is not enough aggregate capacity to cover demand, the equilibrium price equals consumers' valuation and both firms sell at capacity. For the remaining demand realizations, pure strategy equilibria fail to exist given that either (i) firms want to price slightly below the rival to sell at capacity or (ii) want to serve the residual demand at consumers' reservation price. For a given demand realization in Region II, there exists a unique mixed strategy equilibrium such that the two firms mix over a common support, with a lower bound $p$ strictly above zero and an upper bound equal to one, the consumers' reservation price. The equilibrium distribution of prices will depend on both the aggregate capacity and the distribution of capacities. In particular, the larger is $K$ the nearer is $p$ to one; also, for the same value of $K$, the smaller is $k^{-}$the nearer is $p$ to one. Since the large firm plays a mass point at the upper bound, and since the small firm is pricing below that level with probability one, the large firm's expected profits are the same as if it maximized its expected profits over its residual demand.

\subsection{Capacity investment stage}

We can use the above derivation to first consider the deterministic environment, which will serve as a benchmark for the rest of the game. The following proposition characterizes equilibrium capacity choices in the game with certain demand.

Proposition 2. (Cripps and Ireland, 1988) Assume that the mass of buyers is known to be $\theta$. Every profile of firms' capacities with $k^{+} \in\left[\frac{\theta}{2}, \frac{\theta}{2} c\right]$ and $k^{-}=\theta-k^{+}$can be sustained by a pure strategy subgame perfect equilibrium. Hence, equilibrium aggregate capacity equals total demand, and there is a symmetric equilibrium as well as a continuum of asymmetric equilibria.

Under demand certainty, there exists a continuum of pure strategy equilibria, in all of which aggregate capacity is equal to

\footnotetext{
${ }^{7}$ We are very grateful to the authors for providing us with a copy of their manuscript.

${ }^{8}$ In more detail, the large firm plays a mass point at the reservation price with probability $1 \frac{k}{\min \left(\theta, k^{+}\right)}$. Hence, the higher $k$, the less likely it is that the large firm prices at the upper bound of the support of firms' mixed strategies.
}

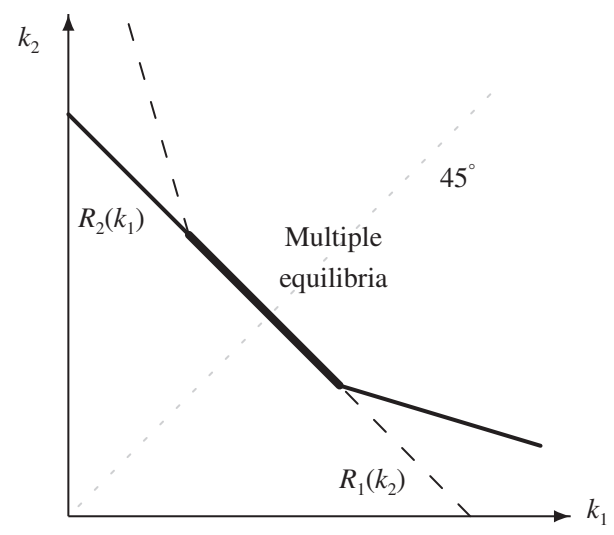

Fig. 1. Best response correspondences under demand certainty.

total demand and prices are set equal to consumers' reservation price. The symmetric capacity pair belongs to the equilibrium set given that firms are discouraged from (a) reducing their capacity, as this would constrain their production without increasing prices; and from (b) increasing their capacity, as this would increase costs with no increase in production (the deviant would become the large firm, so it would serve the residual demand only). Fig. 1 depicts firms' best response correspondences at the capacity stage with certain demand.

Let us now consider the game with demand uncertainty, in which the mass of buyers $\theta$ is randomly determined according to a distribution function $G$ satisfying Assumption 1. Based on Proposition 1, firms' expected profit functions at the investment stage are constructed as follows. For demand realizations in Region I (i.e., below $k^{-}$), expected profits are zero independently of the value of firms' capacities, whereas if $K<1$ then for demand realizations in Region III firms' expected profits are fully determined by their capacity choices. The link between capacity choices and expected profits becomes more complex for demand realizations in Region II, i.e., in the interval $(k, \min \{K, 1\})$. Over this range, the large firm's expected profits do not depend on its own capacity, as these are the same as if it served the residual demand with probability one. In contrast, the small firm's expected profits at the mixed strategy equilibrium depend on its own capacity, for two reasons: first, it constrains its sales when the small firm prices below the rival; and second, it affects the large firm's pricing behavior, ultimately determining its own chances of selling at capacity. Thus, for $i, j=1,2, i \neq j$, denoting $K^{o}=\min \{K, 1\}$, expected profits are

$\pi_{i}\left(k_{i}, k_{j}\right)=\left\{\begin{array}{lll}\pi_{i}\left(k_{i}, k_{j}\right) & \text { if } \quad k_{i} \leq k_{j} \\ \pi_{i}^{+}\left(k_{i}, k_{j}\right) & \text { if } \quad k_{j} \leq k_{i} \leq 1\end{array}\right.$

where $^{9}$

$\pi_{i}^{-}\left(k^{-}, k^{+}\right) \quad \int_{k}^{K^{0}} \frac{k^{-}\left[\theta \quad k^{-}\right]}{\min \left\{\theta, k^{+}\right\}} d G(\theta)+\int_{K^{\circ}}^{1} k^{-} d G(\theta) \quad c k^{-},\left(k^{-}, k^{+}\right) \in[0,1]^{2}: k^{-} \leq k^{+}$,

$\pi_{i}^{+}\left(k^{+}, k^{-}\right)=\int_{k}^{K^{0}}\left[\begin{array}{ll}\theta & k^{-}\end{array}\right] d G(\theta)+\int_{K^{o}}^{1} k^{+} d G(\theta) \quad c k^{+},\left(k^{-}, k^{+}\right) \in[0,1]^{2}: k^{+} \geq k^{-}$.

The expected profit for firm $i$ is a piecewise continuous function made up of the continuous and differentiable functions $\pi_{i}^{-}$and $\pi_{i}^{+}$. The latter is a strictly concave function in own action $k^{+}$whereas the former is strictly concave in $k^{-}$under certain conditions on the

\footnotetext{
${ }^{9}$ Note that for any $k_{j}$, the capacity choice $k_{i}=1$ strictly dominates any capacity choice $k_{i}>1$. Hence, we can restrict the strategy space to the interval $[0,1]$.
} 
density function (sufficient conditions are, for instance, that $g$ be either weakly increasing or concave). The expected profit function $\pi_{i}$ inherits the continuity properties of its two pieces but it does not inherit either their differentiability or their concavity. In particular, the expected profit function has a kink along the diagonal so that it is not everywhere differentiable. To see this let $K \leq 1$. Marginal expected profits from increasing own capacity when the firm is either the small or the large one are given by

$\frac{\partial \pi_{i}}{\partial k}=\int_{k}^{K} \frac{\theta-2 k}{\min \left\{\theta, k^{+}\right\}} d G(\theta)+1-G(K)-c$,

$\frac{\partial \pi_{i}^{+}}{\partial k^{+}}=1-G(K)-c$

so that at symmetric capacity pairs, the right hand derivative is larger than the left hand derivative as, for any G satisfying Assumption 1,

$\left.\lim _{k_{i} \downarrow k} \frac{\partial \pi_{i}^{+}}{\partial k_{i}}\right|_{\left(k_{i}, k\right)}-\left.\lim _{k_{i} \uparrow k} \frac{\partial \pi_{i}}{\partial k_{i}}\right|_{\left(k_{i}, k\right)}=\int_{k}^{2 k} \frac{2 k-\theta}{k} d G(\theta)>0$.

This non differentiability stems from the fact that incentives to undertake marginal increases in capacity in Region II are different if the firm turns out to be the large or the small one. Whereas the large firm gains nothing by expanding its capacity, an increase in the small firm's capacity may lead to either a expected gain or a expected loss depending on the strength of the two effects involved: by increasing its capacity the small firm can expand its production when it prices below the rival; however, as this also makes the large firm more aggressive, the probability that the small firm prices below and hence sells at capacity is reduced.

The non concavity in own action of the expected profit function along the diagonal of the action space results in a jump in firms' best responses, which rules out existence of a symmetric equilibrium in pure strategies. ${ }^{10}$ Moreover, since $\frac{\partial \pi_{i}^{+}(0,0)}{\partial k_{i}}=1-c>0$ and $\frac{\partial \pi_{i}(1,1)}{\partial k_{i}}=$ $-c<0$, it follows that neither is 0 a best reply to 0 , nor is 1 a best reply to 1 . Consequently, at any candidate equilibrium firms must be asymmetric: there will be a large and a small firm despite firms being fully symmetric ex ante. In equilibrium, the firm that ends up being the large one must be choosing a capacity $k^{+}>k^{-}$which maximizes its expected profits. Since $\pi^{+}$is strictly concave in $k^{+}$, the first order condition of the large firm (Eq. (5)) must be satisfied in equilibrium, i.e., $1-G(K)-c=0$.

From this, it follows that aggregate equilibrium capacity is $K=G^{-1}$ $(1-c)$. Aggregate capacity depends on the marginal costs of capacity, $c$, as well as on the shape of the demand distribution function, $G$; in particular, as the degree of convexity of $G$ increases, aggregate equilibrium capacity goes up. Intuitively, firms invest more under more convex distributions as increased convexity implies that higher (lower) demand realizations are more (less) likely. These results are summa rized in the following proposition.

Proposition 3. For any continuous cdf $G$ that satisfies Assumption 1, (i) no symmetric pure strategy Nash equilibrium exists, and (ii) at any candidate equilibrium $\left(k, k^{+}\right)$aggregate capacity is given by $K=k+k^{+}=G^{1}(1-c) \subset(0,1)$.

Further inspection of $\pi_{i}^{+}$shows that capacities are strategic substitutes from the large firm's perspective. In other words, the

\footnotetext{
10 There is a symmetric mixed strategy equilibrium but it is Pareto dominated by any of the two pure-strategy asymmetric equilibria from the point of view of firms' profits. The lower bound in the support of firms' mixed strategies is given by the pure-strategy capacity choice of the small firm, whereas the upper bound is strictly below the purestrategy capacity choice of the large firm.
}

second order cross derivative of the large firm is negative irrespective of how demand is distributed,

$\frac{\partial^{2} \pi_{i}^{+}}{\partial k^{+} \partial k}=-g(K)<0$

The intuition for this result runs as follows. Expected profits by the large firm only depend on its capacity choice if demand is in Region III, as otherwise it serves residual demand only. Since the incidence of demand realizations in Region III is lower the bigger aggregate capacity, the large firm's marginal returns to investment are decreasing in the small firm's capacity.

Matters are not that simple for the small firm, as its second order cross derivative is given by

$\frac{\partial^{2} \pi_{i}}{\partial k \partial k^{+}}=\int_{k^{+}}^{K} \frac{(2 k-\theta)}{\left[k^{+}\right]^{2}} d G(\theta)+g(K)\left(-\frac{k}{k^{+}}\right)$.

The sign of the first term in Eq. (7) is ambiguous, as it depends on the size of the large firm: if $k^{+}$is sufficiently close to $k^{-}$, then the first term is positive, whereas if $k^{+}$is sufficiently large (e.g. if $k^{+}>2 k^{-}$) it is negative. In contrast, the second term in Eq. (7) is clearly negative. The relative strength of these (possibly) opposite signs depends on the shape of the demand distribution. With demand distributions that put more weight on larger demand values, the negative sign of the second term dominates, so that strategic substitutability holds.

We next show that by imposing additional restrictions on the demand distribution function, we can guarantee that capacity choices are strategic substitutes from the small firm's perspective. A sufficient (not necessary) condition for strategic substitutability to hold is that $\theta g(\theta)$ be non decreasing, i.e., $g(\theta)+\theta g^{\prime}(\theta) \geq 0$, as we assume below:

Assumption 2. $\theta g(\theta)$ is non decreasing.

A distribution function that satisfies Assumption 2 is an increasing generalized failure rate distribution (IGFR), i.e., its generalized failure rate, $\frac{\theta g(\theta)}{1 G(\theta)}$, is weakly increasing for all $\theta$ such that $G(\theta)<1 .{ }^{11}$ Note that any convex distribution function satisfies Assumption 2. Moreover, it also holds for some concave distributions, such as the power $G(\theta)=\theta^{a}$ or the exponential $G(\theta)=\frac{1 e^{a \theta}}{1 e^{a}}$, with $0 \leq a \leq 1$.

Assumption 2 has an appealing economic interpretation. Since total revenues when firms sell at capacity are given by $x(1-G(x))$, Assumption 2 is sufficient for ensuring downward sloping mar ginal revenues. This assumption is commonly used in Cournot models to guarantee that best responses are decreasing (see Vives (1999, p. 94)). To see this, let $Q(x)=(1-G(x))$. Then, marginal revenues $Q(x)+x Q^{\prime}(x)$ decrease if Assumption 2 holds as $G^{\prime}(x)+x G^{\prime \prime}(x) \geq 0$ ensures $-2 G^{\prime}(x)-x G^{\prime \prime}(x) \leq 0$.

For any distribution satisfying Assumptions 1 and 2, Proposition 4 shows that existence and uniqueness of equilibrium outcomes can be guaranteed by applying the theory of submodular games. ${ }^{12}$

Proposition 4. For any continuous cdf $G$ that satisfies Assumptions 1 and 2 , the capacity game is a game of strategic substitutes and it has exactly one pair of pure strategy equilibria, $\left(k^{-}, k^{+}\right)$and $\left(k^{+}, k^{-}\right)$, with $k^{-}<k^{+}$.

Fig. 2 depicts firms' best response correspondences at the capacity game when Assumptions 1 and 2 hold. They are symmetric, decreasing, and everywhere continuous except at one point. Since

\footnotetext{
${ }^{11}$ For more on IGFR distributions see Barlow and Proschan (1965).

12 Using the dual single-crossing property instead of submodularity has not allowed us to enlarge the set of distributions for which we can ensure that best-responses are downward-sloping. This is in line with the results in Amir et al. (2010), where one can additionally find further arguments in favor of submodularity.
} 


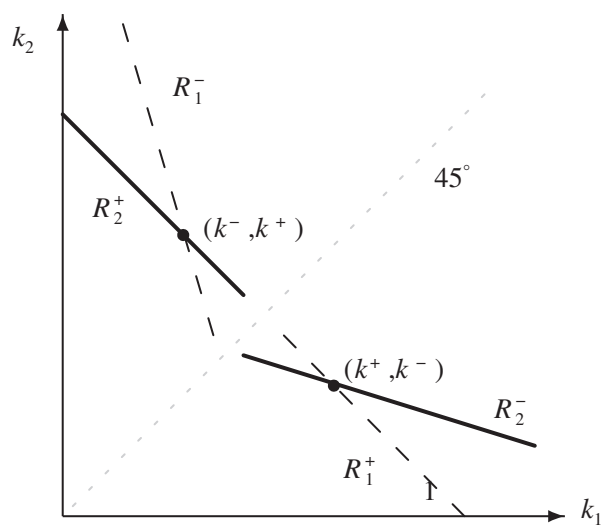

Fig. 2. Best response correspondences for demand distributions $G$ satisfying (A2).

they do not cross the 45 degree line and cross twice, there exist two outcome equivalent asymmetric equilibria. Thus, for each firm $i$, there are two equilibria: one in which firm $i$ is the large firm and one in which firm $i$ is the small one.

Last, let us note that Assumption 2 is a sufficient, not a necessary condition, i.e., even if $G$ does not satisfy Assumption 2, the capacity game may still have a unique pair of asymmetric equilibria. Consider, for instance, the $\operatorname{cdf} G(\theta)=\frac{5}{2} \theta-\frac{13}{6} \theta^{2}+\frac{2}{3} \theta^{3}$ whose density, $g(\theta)=$ $2 \theta^{2}-\frac{13}{3} \theta+\frac{5}{2}$, fails Assumption 2 as $\theta g(\theta)$ decreases for any $\theta \geq 0.4$. Still, for $c=0.15$ there is an equilibrium at $k^{-}=0.139, k^{+}=0.438 .{ }^{13}$

We have characterized the set of subgame perfect pure strategy Nash equilibria for a wide family of demand distributions. Our results are in line with Amir et al.'s (2010), who show that two player symmetric games with payoff nonconcavities and some form of strategic substitutability always possess only asymmetric pure strategy Nash equilibria. In sum, we have provided further support to the claim that asymmetric market structures are a robust prediction in capacity choice models.

\section{Extensions and variations}

In this section we extend the previous analysis by considering alternative formulations regarding capacity cost functions and the nature and timing of demand uncertainty. These extensions cast light on the importance of the assumptions underlying our main model, while allowing to assess the robustness of our conclusions.

\subsection{General capacity cost functions and ex ante asymmetries}

So far we have assumed that firms are ex ante symmetric and that they both have the same constant per unit cost of capacity, $c$. In this section we relax these two assumptions by allowing for nonlinear and possibly asymmetric capacity costs across firms:

Assumption 3. The cost to firm $i$ of installing $k_{i}$ is $C_{i}\left(k_{i}\right), i=1,2$. The function $C_{i}$ is twice continuously differentiable, convex, and satisfies $C_{i}(0)=0$ and $C_{i}^{\prime}\left(k_{i}\right)>0$. To avoid trivialities, $C_{i}^{\prime}(0)<1$, i.e., production at some level is profitable. ${ }^{14}$

The following result generalizes Propositions 3 and 4, for all capacity cost functions satisfying Assumption 3.

Proposition 5. Under Assumption 3, for any continuous cdf $G$ that satisfies Assumption 1, (i) no symmetric pure strategy Nash equilibrium

\footnotetext{
13 The interested reader can find other sufficient conditions for equilibrium existence in the working paper version, De Frutos and Fabra (2007).

${ }^{14}$ We borrow this assumption from Kreps and Scheinkman (1983)'s Assumption 2. It is also assumed by Lepore (2008).
}

exists; if G also satisfies Assumption 2, (ii) the capacity game is a game of strategic substitutes, it has at least one asymmetric pure strategy equilibrium, and exactly two if firms have symmetric cost functions, $C_{i}=C_{j}$.

Whereas Proposition 5 above shows that the nature of equilibria remains qualitatively unchanged, this does not imply that capacity cost functions are irrelevant. To the contrary, the shape and the asymmetries in capacity cost functions affect equilibrium asymme tries across firms, and may have a crucial impact on equilibrium multiplicity. If firms are symmetric but capacity cost functions are nonlinear, there exists exactly one pair of pure strategy outcome equivalent equilibria. However, the shape of the cost function affects the degree of ex post asymmetries and the level of aggregate investment. If cost functions are linear but asymmetric, the two candidate equilibria are no longer outcome equivalent, and one of them may eventually disappear as the difference in costs becomes large enough.

To fix ideas, suppose that firms have constant per unit costs of capacity $c_{1} \leq c \leq c_{2}$. Since the second order and cross derivatives are unchanged, the best replies of the two firms have the same slope as in the symmetric case. However, the best reply of the inefficient (efficient) firm shifts inwards (outwards) given that its marginal profit is now smaller (bigger). This implies that, whenever the difference in costs is not too large, there exist two equilibria that are no longer outcome equivalent. More precisely, since aggregate capacity is determined by the first order condition of the large firm, aggregate capacity under equilibrium $\left(k_{i}, k_{j}^{+}\right)$is $G^{-1}\left(1-c_{j}\right)$; hence, total capacity is larger at the equilibrium at which the efficient firm has the large capacity. However, if the difference in costs is wide enough, best replies no longer cross in the region at which the large firm is the efficient one, so that the unique equilibrium is $\left(k_{1}^{+}, k_{2}^{-}\right)$. Table 1 illustrates these results in a numerical example.

\subsection{Two point distribution}

So far we have assumed that the mass of buyers $\theta$ is distributed according to a continuous function. Let us now assume instead that demand follows a binomial distribution function:

Assumption 4. $\theta$ equals $\theta^{L}$ with probability $\rho \in(0,1)$ and $\theta^{H}$ with probability $1-\rho$, where $0<\theta^{L}<\theta^{H}$.

The following proposition characterizes the subgame perfect equilibrium capacity choices in this case.

Proposition 6. Under Assumption 4, the following is true:

(i) In any subgame perfect pure strategy equilibrium, aggregate capacity is $\theta^{L}$ if $\rho \in(1-c, 1]$ and it is $\theta^{H}$ if $\rho \in[0,1-c)$. In either case, the equilibrium set of this game belongs to the equilibrium set when the mass of buyers is either $\theta^{L}$ or $\theta^{H}$ with certainty. If $\rho=1-c$, any aggregate capacity $K \in\left[\theta^{L}, \theta^{H}\right]$ can be sustained as an equilibrium outcome.

(ii) For all $\rho$, there exists a continuum of asymmetric pure strategy equilibria in capacity choices.

Table 1

Equilibrium capacities as a function of the per-unit capacity cost of the inefficient firm $c_{2}$, for $c_{1}=0.1$; uniformly distributed demand.

\begin{tabular}{|c|c|c|c|c|c|c|c|}
\hline \multicolumn{4}{|c|}{ Equilibrium $\left(k_{1}, k_{2}^{+}\right)$} & \multicolumn{4}{|c|}{ Equilibrium $\left(k_{1}^{+}, k_{2}\right)$} \\
\hline & $c_{2}=0.1$ & $c_{2}=0.2$ & $c_{2}=0.4$ & & $c_{2}=0.1$ & $c_{2}=0.2$ & $c_{2}=0.4$ \\
\hline$k_{1}$ & 0.27 & 0.32 & - & $k_{1}^{+}$ & 0.63 & 0.69 & 0.77 \\
\hline$k_{2}^{+}$ & 0.63 & 0.48 & - & $k_{2}$ & 0.27 & 0.21 & 0.13 \\
\hline$K$ & 0.9 & 0.8 & - & $K$ & 0.9 & 0.9 & 0.9 \\
\hline
\end{tabular}


(iii) There exists $\rho \in\left(\frac{1 c}{2}, 1-c\right)$ such that symmetric equilibria in capacity choices exist if and only if $\rho \notin(\rho, 1-c)$.

The first part of Proposition 6 shows that if $1-\rho \neq c$, aggregate equilibrium capacity is either $\theta^{L}$ or $\theta^{H}$. Aggregate capacity cannot be below $\theta^{L}$, as expanding capacity up to $\theta^{L}$ would allow firms to increase production without reducing prices. Similarly, aggregate capacity cannot exceed $\theta^{H}$, as the large firm would never sell at capacity and could thus increase its expected profits by reducing capacity without constraining its production. The issue of whether firms invest $\theta^{L}$ or $\theta^{H}$ depends on the comparison between the marginal returns to investment when demand is high, $1-\rho$, and the marginal costs of the extra capacity, $c$. If $1-\rho<c$, the marginal costs exceed the marginal gains of increasing capacity, so that firms invest just enough so as to cover demand in the low state. Alternatively, if $1-\rho>c$, the marginal gains exceed the marginal costs and firms expand capacity in order to fully cover demand in the high state. This implies that aggregate capacity is the same as if demand was known to be either low or high with certainty whenever the probability of the low demand state is either sufficiently large (i.e., $\rho \in(1-c, 1]$ ) or sufficiently small (i.e., $\rho \in[0,1-c)$ ), respectively. In contrast, if $1-\rho=c$, any capacity expansion aimed at increasing production when demand is high yields a zero marginal profit. This implies that any capacity pair resulting in $K \in\left[\theta^{L}, \theta^{H}\right]$ can be sustained by a subgame perfect equilibrium of the game.

Similar to the certain demand case, the investment game under a two point distribution generates a continuum of pure strategy equilibria, ${ }^{15}$ some of which involve asymmetric capacity choices. However, unlike the certain demand case, the existence of a symmetric equilibrium is not guaranteed whenever it generates excess capacity with positive probability. If that is the case, one firm may find it profitable to marginally reduce its capacity to become the small firm, not only because it implies lower capital costs, but also because it may induce less aggressive pricing by its rival.

In order to understand the above result, let us analyze the profitability of deviating from the two candidate symmetric equilibria, $\left(\theta^{L} / 2, \theta^{L} / 2\right)$ and $\left(\theta^{H} / 2, \theta^{H} / 2\right)$. Consider first $\left(\theta^{L} / 2, \theta^{L} / 2\right)$. Following part (i) of the Proposition, for such an equilibrium to exist the probability of the low demand state must be sufficiently large, i.e., $\rho \in[1-c, 1]$. For all values of $\rho$ in this range, the candidate symmetric equilibrium exists since (a) reducing capacity below $\theta^{L} / 2$ would further constrain production without increasing prices, and (b) the marginal costs of expanding capacity, $c$, would exceed the associated marginal gain, $1-\rho$.

Consider now the alternative symmetric candidate equilibrium, $\left(\theta^{H} / 2, \theta^{H} / 2\right)$. For such an equilibrium to exist the probability of the low demand state must be sufficiently small, i.e., $\rho \in[0,1-c]$. Since aggregate equilibrium capacity equals $\theta^{H}$, capacity is fully utilized and prices are at its maximum only when demand is high. Thus, part (a) of the above reasoning no longer holds. In particular, since for low demand there is excess capacity and prices are below their reservation level, a marginal reduction in capacity below $\theta^{H} / 2$ would not constrain the deviant's production and it would lead to both capacity savings and higher prices. Such deviation gains have to be balanced against the marginal loss due to the output reduction when demand is high, an issue which ultimately depends on the relative incidence of the low and high states. If the probability of the low demand state is sufficiently large, i.e., $\rho \in(\rho, 1-c)$, the deviation gains outweigh the deviation losses, thus ruling out existence of a symmetric equilibrium.

Finally, the impact of demand uncertainty on equilibrium multiplicity depends on whether demand is price responsive or not: whereas in

\footnotetext{
${ }^{15}$ This result is specific of the two-point distribution. For instance, if demand can take three equally likely values, then a unique equilibrium outcome emerges in which firms choose asymmetric capacities.
}

Reynolds and Wilson (2000, Theorem 2) demand uncertainty (weakly) increases the number of equilibria, we have shown that it reduces equilibrium multiplicity when demand is price inelastic.

\subsection{Demand uncertainty at the pricing stage}

So far we have assumed that firms observe the demand realization before setting prices. Instead, let us now assume that demand is also uncertain at the pricing stage. Since this assumption changes the pricing stage, we first provide equilibrium characterization at the second stage of the game.

Proposition 7. If capacities and prices are chosen prior to the actual realization of $\theta$, then for any continuous cdf $G$ that satisfies Assumption 1, there exists a unique equilibrium for given $k^{-} \leq k^{+}$, that satisfies the following:

(i) If $k^{-} \geq 1$, at the unique pure strategy equilibrium both firms set prices equal to marginal cost and make zero expected profits.

(ii) If $k^{-}<1$, a pure strategy equilibrium fails to exist. In the unique mixed strategy equilibrium, ${ }^{16}$ firms choose prices in a common support, whose lower bound is strictly above marginal cost and whose upper bound is 1.

If the small firm chooses to be large enough so that its capacity always exceeds the largest possible demand realization, firms set prices equal to marginal costs. However, and precisely for this reason, this case would never arise as a subgame perfect equilibrium of the two stage game. If the small firm is always capacity constrained to serve the market alone, the equilibrium differs substantially from the case in which demand is known with certainty before prices are set. In particular, the game has no pure strategy equilibria. At the unique mixed strategy equilibrium, both firms charge prices in a common support, with a lower bound strictly above (zero) marginal costs, and an upper bound equal to consumers' reservation price; unlike the small firm, the large firm plays a mass point at the upper bound.

Based on Proposition 7, we can construct the firms' expected profit functions at the investment stage as a function of their capacity choices. For $i, j=1,2, i \neq j$,

$\pi_{i}\left(k_{i}, k_{j}\right)=\left\{\begin{array}{lll}\pi_{i}\left(k_{i}, k_{j}\right) & \text { if } & k_{i} \leq k_{j} \\ \pi_{i}^{+}\left(k_{i}, k_{j}\right) & \text { if } & k_{i} \leq k_{j}\end{array}\right.$,

where, setting $h(z)=\int_{0}^{1} \min \{\theta, z\} d G(\theta)$,

$\pi_{i}^{-}\left(k^{-}, k^{+}\right) \quad \frac{h\left(k^{-}\right)}{h\left(k^{+}\right)} \int_{k}^{1} \min \left\{\theta \quad k^{-}, k^{+}\right\} d G(\theta) \quad c k^{-},\left(k^{-}, k^{+}\right) \in[0,1]^{2}: k^{-} \leq k^{+}$,

$\pi_{i}^{+}\left(k^{+}, k^{-}\right)=\int_{k}^{1} \min \left\{\theta-k^{-}, k^{+}\right\} d G(\theta)-c k^{+},\left(k^{-}, k^{+}\right) \in[0,1]^{2}: k^{+} \geq k^{-}$

The expected profit for firm $i$ is a piecewise continuous function made up of the continuous and differentiable functions $\pi_{i}^{-}$and $\pi_{i}^{+}$. The latter is strictly concave in $k^{+}$whereas the former is strictly concave in $k^{-}$under certain conditions on the density function (e.g. a weakly increasing $g$ suffices for $\pi_{i}^{-}$to be strictly concave in $k^{-}$).

For the given capacities, the large firm's expected profits, Eq. (10), are the same as in the game in which firms face no uncertainty at the pricing stage, Eq. (3). In either case, the large firm earns the same

\footnotetext{
${ }^{16}$ If $F$ and $F^{+}$denote the equilibrium mixed-strategy of the small and the large firm, respectively, then $F(p) \geq F^{+}(p)$ follows. Gal-Or (1984) focuses on the symmetric mixed strategy equilibrium. The mixed strategy equilibrium is symmetric only if $k=k^{+}=k$ (see Appendix A for details).
} 
profits as if it were as a monopolist over the residual demand. In contrast, the smaller firm's expected profits do depend on the timing of demand realizations. For given capacity choices, the expected revenue of the small firm is now a fraction of the larger firm's expected revenue. This fraction is given by the ratio of the expected revenues of a monopolist with capacity $k^{-}$over those of a monopolist with capacity $k^{+}$.

The expected profit function preserves the main feature that accounts for the non existence of a symmetric equilibrium in capacity choices: marginal returns to investment differ across firms. Formally, the marginal expected profits from increasing own capacity, condi tionally on being the small or the large firm, are given by

$$
\frac{\partial \pi_{i}}{\partial k}=\frac{(1-G(k)) \int_{k}^{1} \min \left\{\theta-k, k^{+}\right\} d G(\theta)-h(k) \int_{k}^{K} d G(\theta)}{h\left(k^{+}\right)}-c,
$$

$\frac{\partial \pi_{i}^{+}}{\partial k^{+}}=1-G(K)-c$.

Consequently, marginal returns to investment jump around symmetric capacity pairs,

$\left.\left.\lim _{k_{i} \downarrow k} \frac{\partial \pi_{i}^{+}}{\partial k_{i}}\right|_{\left(k_{i}, k\right)} \lim _{k_{i} \uparrow k} \frac{\partial \pi_{i}^{-}}{\partial k_{i}}\right|_{\left(k_{i}, k\right)}=\frac{1 G(k)}{h(k)}\left(\int_{0}^{k} \theta d G(\theta)+\int_{k}^{2 k}(2 k \quad \theta) d G(\theta)\right)>0$,

ruling out existence of a symmetric equilibrium in capacity choices.

Since at any candidate equilibrium there is a large and a small firm, the former must invest $k^{+}$to maximize its expected profits. As $\pi_{i}^{+}$is strictly concave in $k^{+}$, any candidate equilibrium must solve the large firm's first order condition, (12). It follows that aggregate capacity is given by $K=G^{-1}(1-c)$. The analogous to Proposition 3 trivially follows.

Proposition 8. If capacities and prices are chosen prior to the actual realization of $\theta$, then for any continuous cdf $G$ that satisfies Assumption 1 , (i) no symmetric pure strategy Nash equilibrium exists, and (ii) at any equilibrium candidate $\left(k, k^{+}\right)$aggregate capacity is given by $K=k+k^{+}=G^{1}(1-c) \subset(0,1)$.

Regarding the nature of the strategic interaction, capacity choices are always strategic substitutes from the large firm's perspective. In contrast, this may not always hold for the small firm. To see this, let us re write the small firm's marginal returns to investment as follows:

$$
\frac{\partial \pi_{i}^{-}}{\partial k^{-}} \quad \frac{h\left(k^{-}\right)}{h\left(k^{+}\right)}(1 \quad G(K)) \frac{h(k)-\int_{k}^{1} \min \left\{\theta-k, k^{+}\right\} d G(\theta)}{h\left(k^{+}\right)}(1-G(k))-c .
$$

Whereas the first term is always decreasing in $k^{+}$, the second term need not be. Indeed, the second term is increasing in $k^{+}$whenever the numerator, $h(k)-\int_{k}^{1} \min \left\{\theta-k, k^{+}\right\} d G(\theta)$, is positive. This is always the case unless $k^{-}$is sufficiently small. The relative weights of these two possibly opposite effects determine whether capacity choices are strategic substitutes or strategic complements from the small firm's perspective. A sufficient (not necessary) condition for strategic substitutability to hold is that the density function be non decreasing, as stated in the following assumption:

Assumption 5. $g$ is non decreasing.

Note that Assumption 5 is stronger than Assumption 2. Moreover, there are demand distributions for which we can guarantee that capacity choices are strategic substitutes when firms face uncertainty at the investment stage only, while they are not when firms face demand uncertainty at both stages. ${ }^{17}$ The analogous to Proposition 4 reads as follows.

Proposition 9. If capacities and prices are chosen prior to the actual realization of $\theta$, then for any continuous $c d f G$ that satisfies Assumptions 1 and 5, the capacity game is a game of strategic substitutes and it has exactly one pair of pure strategy equilibria $\left(k^{-}, k^{+}\right)$and $\left(k^{+}, k^{-}\right)$.

As we have shown, the nature of the equilibria as well as the level of total investment is the same under both timing of demand uncertainty. However, prices and firms' expected profits need not be the same. In order to illustrate this, we focus on the case in which demand is uniformly distributed.

Lemma 1. Assume that demand is uniformly distributed on the unit interval. Prices and expected profits are higher if prices are chosen prior to the actual realization of $\theta$ than if they are chosen after the actual realization of $\theta$.

The timing of demand uncertainty alters the way in which total capacity is distributed among firms, thereby affecting market concentration. At least when demand is uniformly distributed, firms' capacities are more asymmetric when firms face uncertain demand at the pricing stage also. This leads to greater expected profits, and hence higher consumer prices, while total welfare remains the same (recall that welfare only depends on aggregate investment). It follows that the most favorable outcome from consumers' point of view is achieved when firms know he actual mass of consumers prior to competing in the product market.

\section{Comparison with other games}

We conclude the paper by comparing the equilibria of the two stage game, as analyzed in Section 2, with those in two benchmark games: the certainty equivalent game, and the Cournot game with and without demand uncertainty.

\subsection{Certainty equivalent game}

We first consider the certainty equivalent game, i.e., the game in which demand is known to be equal to the expected demand in the uncertain game, i.e., to $E[\theta]=\int_{0}^{1} \theta d G(\theta)$.

Lemma 2. Comparison of the subgame perfect equilibrium outcomes in the certainty equivalent game versus the game with demand uncertainty (regardless of whether firms face demand uncertainty at both stages or only at the investment stage) shows the following:

(i) Aggregate capacity is larger under demand uncertainty if and only if $c \in(0, \widehat{c})$, where $\widehat{c}$ is implicitly defined by $G^{1}(1-\widehat{c})=E[\theta]$.

(ii) Both with certain and uncertain demand, aggregate investment is welfare maximizing. Nevertheless, the level of total welfare at the First Best is lower under demand uncertainty.

(iii) Prices are lower and consumer surplus is higher under demand uncertainty.

(iv) The game with demand uncertainty generates price dispersion, whereas its certainty equivalent game does not.

Under demand uncertainty, a marginal increase in the large firm's capacity allows it to sell more output at the reservation price whenever demand exceeds aggregate capacity, but it implies an additional investment cost, $c$. Hence, an increase in $c$ reduces investment, and may

\footnotetext{
${ }^{17}$ For instance, for the family of power functions $G(\theta)=\theta^{a}, a \in(0,1)$, which satisfy Assumption 2 but not Assumption 5, the cross-partial derivative of the small firm is positive at any pair $\left(k, k^{+}\right)$for $k$ sufficiently small. Hence, within this family of distribution functions, capacity choices are not always strategic substitutes if demand is uncertain at both the investment and pricing stages. In contrast, they are strategic substitutes if demand is uncertain at the first stage only.
} 
ultimately lead to very low investment levels as $c$ approaches consumers' reservation price. In contrast, under demand certainty, firms invest just enough so as to cover demand irrespective of the unit cost of capacity (as long as it does not exceed consumers' reservation value). Therefore, demand uncertainty generates more investment as compared to the certainty equivalent game if and only if $c$ is sufficiently low with respect to expected demand.

Regardless of whether demand is certain or uncertain, aggregate investment is the same as the one a social planner would choose in order to maximize total welfare. Social and private gains from increasing capacity coincide since the marginal capacity is used when aggregate capacity is fully utilized, in which case it receives consumers' reservation price. This does not mean however that welfare levels are the same under both games. Trivially, the fact that $K<1$ implies that in the uncertain game total surplus is not maximal for demand realizations in $[K, 1]$. In contrast, under the certainty equivalent game, there is no demand rationing nor unused capacity, so that total welfare is maximized.

Regarding prices and consumer surplus, the ranking goes in the opposite direction. Under demand uncertainty, the emergence of idle capacity for some demand realizations drives prices below the reservation price, allowing consumers to retain a positive share of total surplus. This contrasts with the certainty equivalent game, in which prices are set equal to consumers' reservation value, thus driving consumer surplus to zero. ${ }^{18}$

Last, as argued by Gal Or (1984), our results confirm that a rationale for price variability over time can be based upon uncertain demand: at the equilibrium capacities of the uncertain demand game, mixed strategy pricing generates price dispersion; this is unlike its certainty equivalent game, in which prices remain at the constant reservation price.

\subsection{Cournot game}

There is a long tradition of comparing the sequential capacity price game and the Cournot game, which dates back to Kreps and Scheink man's seminal paper. For a duopoly facing downward sloping and certain demand, Kreps and Scheinkman were the first to show that the unique equilibrium of the sequential capacity price game yields the Cournot outcome. By introducing demand uncertainty into Kreps and Scheinkman's model, Reynolds and Wilson (2000) showed that the equivalence between these two games need not always hold. In particular, for some parameter values, the equilibrium capacity choices of the two stage game are asymmetric, and hence do not belong to the set of Cournot equilibria, as these are all symmetric. ${ }^{19}$

As we show next, Kreps and Scheinkman's result is more robust to introducing demand uncertainty if demand is price inelastic than if it is downward sloping, in the following sense: the equilibrium capacity choices of the two stage game are always contained in the set of Cournot equilibria, regardless of whether demand is certain or not. However, this does not imply that both games are fully outcome equivalent as, under demand uncertainty, the two stage game leads to more competitive pricing than the Cournot game.

At the Cournot equilibrium, the strategy of firm $i$ is a choice of output that maximizes its profits given its rival's output choice,

$\pi_{i}\left(q_{i}, q_{j}\right)=\int_{0}^{1} \min \left\{q_{i}, \theta-q_{j}\right\} d G(\theta)-c q_{i}, \quad i=1,2$.

\footnotetext{
${ }^{18}$ When firms choose output levels in the second stage rather than prices, as in Gabszewicz and Poddar (1997), expected price and output under uncertain demand coincide with the price and output corresponding to the certainty equivalent case. Here we show that this is no longer true when firms compete in prices in the second stage.

19 A sufficient condition for the equivalence between the two games is provided by Lepore (2008, see Theorem 3 ) who shows that the unique equilibrium of the capacity game is the largest uncertain Cournot equilibrium (UCE, for short), if at the UCE capacity pricing is at the Cournot region with probability one.
}

Because demand is perfectly inelastic, the Cournot price always equals the reservation price (normalized to 1 ), as it is the market clearing price regardless of the choice of output of the firms.

With certain demand, there is a continuum of Cournot equilibria, in all of which firms' aggregate output equals total demand (Cripps and Ireland (1988)): any increase in production would remain unsold, whereas any output reduction would imply a profit loss of $1-c$. This equilibrium outcome is efficient and allows firms to earn monopoly profits. Comparing this result to the one in Proposition 2 shows that, in a deterministic environment, the subgame perfect equilibria of the sequential capacity price game are contained in the set of Cournot equilibria.

To explore whether this equivalence remains true under demand uncertainty, we next characterize equilibria at the uncertain Cournot game.

Lemma 3. For any continuous cdf $G$ that satisfies Assumption 1, there are multiple equilibria in the Cournot game with uncertain demand, in all of which aggregate production is $G^{1}(1-c)$.

As far as capacity choices are concerned, the equivalence between the Cournot game and the sequential capacity price game is preserved. More explicitly, the equilibrium capacity choices of the sequential capacity price game are contained in the set of Cournot equilibria, and both games lead to the same aggregate equilibrium investment. However, firms' total equilibrium profits in the Cournot game are higher than those in the sequential capacity price game. Whereas in the former, firms always receive the reservation price and hence earn monopoly profits; in the latter, firms price below that level with probability $1-c$. Last, the two games also differ in an empirically testable prediction: whereas the sequential game generates price dispersion, the Cournot game does not.

\section{Conclusions}

We have characterized the set of subgame perfect Nash equilibria in a game in which firms take pure strategy investment decisions and then engage in price competition. We have shown that the introduction of demand uncertainty has important implications on equilibrium investment choices, as it may rule out the existence of symmetric equilibria, leading to asymmetric capacity choices, regard less of whether firms face demand uncertainty at both the investment and pricing stages or only at the former. Firms become asymmetric in size in an attempt to generate price distributions which yield higher expected prices for a wider range of demand realizations. By doing so, they avoid both competitive prices if demand turns to be below the small capacity (Region I), as well as to avoid more competitive price distributions if demand turns to be below aggregate capacity (Region II). Comparison with the Cournot game shows that under demand uncertainty, subgame perfect equilibrium capacity choices of the two stage game are contained in the set of Cournot equilibria. However, unlike the Cournot game, the capacity price game leads to prices below the reservation price and generates price dispersion.

Our formulation contributes to the existing literature on capacity choices and imperfect competition under demand uncertainty in several respects. First, in contrast to the papers that assume Cournot competition, our approach conforms to the widely accepted view that firms compete in prices subject to capacity constraints. Second, as compared to the papers that analyze investment decisions followed by Bertrand competition, our approach is appealing in terms of tractability, as it provides a characterization of pure strategy equilibria for general demand distributions and cost functions, allowing to make use of powerful results within the theory of submodular games. Last, our model is able to generate relevant predictions regarding market structure and the sensitivity of investment decisions and pricing behavior to measurable variables, such as the shape of the demand 
distribution function or the capacity cost function, and market characteristics, such as the timing of demand uncertainty.

Our analysis may shed light on investment incentives and endogenous market structure in a large set of industries characterized by long lived assets that involve large sunk cost investments, imperfect competition and demand fluctuations. These features are common to most important industries producing commodities, such as steel, chemicals, cement, or electricity, to name just a few, as well as to service industries, such as the Internet.

\section{Acknowledgment}

We thank the editor Volker Nocke and one anonymous referee for their insightful comments. Comments by Diego Moreno and seminar participants at the Simposio de Análisis Económico (Granada) are gratefully acknowledged. The first and second authors acknowledge the financial support awarded by the Spanish Ministry of Education and Science, Project SEJ2007 67436, and the Spanish Ministry of Science and Innovation, Project 2010/02481/001, respectively.

\section{Appendix A}

\section{A.1. The model}

Proof of Proposition 4. We first show that both $\pi^{-}$and $\pi^{+}$are submodular by analyzing the sign of the second order cross derivatives. The second order cross derivatives conditionally on being the small or the large firm are given by

$$
\begin{aligned}
\frac{\partial^{2} \pi_{i}}{\partial k \partial k^{+}} & =-\frac{1}{k^{+}}\left(\int_{k^{+}}^{K} \frac{(\theta-2 k)}{k^{+}} d G(\theta)+g(K) k\right), \text { and } \\
\frac{\partial^{2} \pi_{i}^{+}}{\partial k^{+} \partial k} & =-g(K) .
\end{aligned}
$$

The second order cross derivative of the large firm is negative for any $G$ as $g(K)>0$. Similarly, for any $G$ the second order cross derivative of the small firm is negative at any pair $\left(k^{-}, k^{+}\right)$such that $k^{+} \geq 2 k^{-}$as $\int_{k^{+}}^{K} \frac{(\theta 2 k)}{k^{+}} d G(\theta)>0$. If $G$ satisfies Assumptions 1 and 2 the result does also hold for pairs $\left(k^{-}, k^{+}\right)$such that $k^{-} \leq k^{+}<2 k^{-}$. Note that integration by parts allows to rewrite $\frac{\partial^{2} \pi_{i}}{\partial k \partial k^{+}}$as $-\frac{1}{k^{+}} H\left(k, k^{+}\right)$, where

$$
\begin{aligned}
H\left(k, k^{+}\right) & =\int_{k^{+}}^{K} \frac{(\theta-2 k)}{k^{+}} d G(\theta)+g(K) k \\
& =\frac{1}{k^{+}} \int_{k^{+}}^{K}\left(G(K)+k^{+} g(K)-\left(G(\theta)+\left(2 k-k^{+}\right) g(\theta)\right)\right) d \theta \\
& \geq \frac{1}{k^{+}} \int_{k^{+}}^{K}\left(G(K)+k^{+} g(K)-\left(G(\theta)+k^{+} g(\theta)\right)\right) d \theta,
\end{aligned}
$$

where the second equality follows from straightforward computa tions after integration by parts of $\theta g(\theta)$, and the inequality from the fact that $2 k^{-}-k^{+} \leq k^{+}$. A sufficient condition for the integral above to be positive is that $G(\theta)+k^{+} g(\theta)$ be an increasing function, which holds trivially if $g(\theta)+\theta g^{\prime}(\theta) \geq 0$, i.e., under Assumptions 1 and 2 .

Now existence of at least one pair of asymmetric equilibria follows from Theorem 3.1. in Amir et al. (2010) as (i) $\pi^{-}$and $\pi^{+}$are submodular, (ii) $\left.\lim _{k_{i} \downarrow k} \frac{\partial \pi_{i}^{+}}{\partial k_{i}}\right|_{\left(k_{i}, k\right)}-\left.\lim _{k_{i} \uparrow k} \frac{\partial \pi_{i}}{\partial k_{i}}\right|_{\left(k_{i}, k\right)}=\int_{k}^{2 k} \frac{2 k \theta}{k} d G(\theta)>0$ and iii) $\left.\frac{\partial \pi_{i}}{\partial k_{i}}\right|_{(1,1)}<0$ and $\left.\frac{\partial \pi_{i}^{+}}{\partial k_{i}}\right|_{(0,0)}>0$, so that $(0,0)$ and $(1,1)$ can be ruled out as equilibrium outcomes.

Regarding uniqueness the result follows from the fact that the slope of the best correspondence function of the small firm is smaller than the one corresponding to the large firm so that they can cross only once, i.e., $\left|\frac{\partial^{2} \pi_{i}}{\partial k \quad \partial k^{+}}\right|-\left|\frac{\partial^{2} \pi_{i}^{+}}{\partial k^{+} \partial k}\right|<0$ holds.
Since under Assumptions 1 and 2 both cross derivatives are negative, we have

$$
\left|\frac{\partial^{2} \pi_{i}}{\partial k \partial k^{+}}\right|-\left|\frac{\partial^{2} \pi_{i}^{+}}{\partial k^{+} \partial k}\right|=\frac{1}{k^{+}} \int_{k^{+}}^{K} \frac{(\theta-2 k)}{k^{+}} d G(\theta)-g(K)\left(\frac{k^{+}-k}{k^{+}}\right) .
$$

Furthermore,

$$
\frac{1}{k^{+}} \int_{k^{+}}^{K} \frac{(\theta-2 k)}{k^{+}} d G(\theta)<\frac{1}{k^{+}} g(K)\left(\frac{k^{+}-k}{k^{+}}\right) k<g(K)\left(\frac{k^{+}-k}{k^{+}}\right),
$$

where the first inequality follows from the fact that $\theta g(\theta)$ is an increasing function and the second one from the fact that $k^{-} / k^{+}<1$. It hence follows that $\left|\frac{\partial^{2} \pi_{i}}{\partial k \quad \partial k^{+}}\right|-\left|\frac{\partial^{2} \pi_{i}^{+}}{\partial k^{+} \partial k}\right|<0$, as claimed. Q.E.D.

\section{A.2. Extensions and variations}

Proof of Proposition 5. (i) Marginal expected profits from increasing own capacity are given by Eqs. (4) and (5), except for the last term which now becomes $-C_{i}^{\prime}\left(k_{i}\right)$. For any $C_{i}\left(k_{i}\right)$, it remains true that at symmetric capacity pairs $(k, k)$, with $k \in(0,1)$, the difference between the right hand and the left hand derivatives is positives as $C_{i}^{\prime}(k)$ simply cancels. Hence, (6) remains the same. Moreover, $\left.\frac{\partial \pi_{i}^{+}}{\partial k_{i}}\right|_{(0,0)}=$ $1-C_{i}^{\prime}(0)>0$ and $\left.\frac{\partial \pi_{i}}{\partial k_{i}}\right|_{(1,1)}=-C_{i}^{\prime}(1)<0$, so that $(0,0)$ and $(1,1)$ can be ruled out as equilibrium outcomes. This rules out symmetric equilibria.

(ii) Given that $C_{i}\left(k_{i}\right)$ does not depend on $k_{j}$, the second order cross derivatives are the same as in Proposition 4 , and are hence negative whenever Assumption 2 holds. Together with (i), this assures that $\pi^{-}$and $\pi^{+}$are submodular so that equilibrium existence is guaranteed. If firms are symmetric, uniqueness also follows from the fact that the cross derivatives remain unchanged as in Proposition 4. Q.E.D.

Proof of Proposition 6. (i) The proof is by way of contradiction. Assume that there is an equilibrium at which $K \notin\left\{\theta^{L}, \theta^{H}\right\}$ for some $\rho \neq 1-c$. W.l.o.g. index firms such that $k_{1} \leq k_{2}$. Since $\pi_{1}\left(k_{1}, k_{2}\right)=$ $(1-c) k_{1}$ for all $k_{1} \leq \theta^{L}-k_{2}$, it is trivial to see that $K<\theta^{L}$ cannot be an equilibrium outcome as firm 1 would find it profitable to increase its capacity to $\theta^{L}-k_{2}$. Similarly, $K>\theta^{H}$ cannot be an equilibrium either as firm 2 would prefer to decrease its capacity to $\theta^{H}-k_{1}$. Note that the large firm sells at most the residual demand $\rho\left(\theta^{L}-k_{1}\right)+(1-\rho)\left(\theta^{H}-k_{1}\right)$, which is independent of its own capacity, but has to pay $c$ for any extra capacity. Consequently, $K \in\left[\theta^{L}, \theta^{H}\right]$. Assume now that $K \in\left(\theta^{L}, \theta^{H}\right]$ and $\rho \in(1-c, 1]$. Since firm 2's expected profits for $k_{2} \in\left[k_{1}, \theta^{H}-k_{1}\right]$ are at most $\rho\left(\theta^{L}-k_{1}\right)+(1-\rho-c) k_{2}$ and these are strictly decreasing in $k_{2}$ (note that marginal returns to capacity expansions, $1-\rho-c$, are negative), firm 2 can increase its expected profits by investing $\theta^{L}-k_{1}$, contradicting that $K \in\left(\theta^{L}, \theta^{H}\right]$ is an equilibrium. Finally, assume $K \in\left[\theta^{L}, \theta^{H}\right)$ and $\rho<1-c$. Firm 2's expected profits for $k_{2} \in\left[k_{1}, \theta^{H}-k_{1}\right]$ are strictly increasing in $k_{2}$ (since we now have $1-\rho-c>0$ ) so that firm 2 is better off investing $\theta^{H}-k_{1}$ rather than any smaller quantity, again a contradiction. Finally, if $1-\rho=c$, the probability of the high demand state equals the marginal cost of capacity. Hence, any capacity expansion aimed to be used under high demand yields no marginal profit. Consequently, if $k_{1}=\theta^{L} / 2$, firm 2 is indifferent between setting $k_{2}=\theta^{L} / 2$ or any $k_{2} \in\left[\theta^{L} / 2, \theta^{H}-\theta^{L} / 2\right]$. This implies that any capacity pairs resulting in $K \in\left[\theta^{L}, \theta^{H}\right]$ can be sustained as an equilibrium of the game. 
(ii) and (iii). We first show that if $\rho \in(1-c, 1]$ equilibria are pairs $\left(\theta^{L}-k^{+}, k^{+}\right)$with $k^{+} \in\left[\frac{\theta^{L}}{2}, \frac{\theta^{L} \rho}{1+\rho \quad c}\right]$ so that a symmetric equilibrium exists as well as a continuum of asymmetric equilibria. Focus on firm 1. By i) we can concentrate on choices such $\theta^{L}-k_{2} \leq k_{1} \leq k_{2}$. Appealing to Proposition 1 , firm 1 expected profits are given by $\rho \frac{k_{1}}{k_{2}}\left(\theta^{L}-k_{1}\right)+$ $(1-\rho) k_{1}-c k_{1}$, which are maximized at $k_{1}^{*}\left(k_{2}\right)=\frac{1}{2}\left[\theta^{L}+k_{2} \frac{1}{\rho} \rho\right]$ if it is larger than $\theta^{L}-k_{2}$ and at $\theta^{L}-k_{2}$ otherwise. Since $k_{1}^{*}\left(k_{2}\right)<\theta^{L}-k_{2}$ for any $k_{2} \leq \theta^{L} \frac{\rho}{1+\rho c}$, the best reply is $\theta^{L}-k_{2}$ for $0 \leq k_{2} \leq \theta^{L} \frac{\rho}{1+\rho c}$ and it is $k_{1}^{*}\left(k_{2}\right)$ for $k_{2} \in\left[\theta^{L} \frac{\rho}{1+\rho c}, \theta^{L}\right]$. As $\frac{\rho}{1+\rho c}>\frac{1}{2}$, a symmetric equilibrium always exist together with a continuum of asymmetric equilibria, which shows our claim. Furthermore, as $\rho \rightarrow 1-c$ the only equilibrium is the symmetric one.

Let us now assume $\rho \in[0,1-c]$. Since any candidate equilibrium must satisfy $K=\theta^{H}$, we can focus on firm 1 's best reply to $k_{2} \geq \frac{\theta^{H}}{2}$. We must distinguish two cases: $\frac{\theta^{H}}{2}>\theta^{L}$ and $\frac{\theta^{H}}{2} \leq \theta^{L}$, i.e., if we let $\Delta=\frac{\theta^{H}}{\theta^{L}}$, these two cases are $\Delta>2$ and $\Delta \leq 2$. In what follows, for each value of $\Delta$, we first show that a symmetric equilibrium does not always exist and then characterize the set of asymmetric equilibria.

We first show that a symmetric equilibrium exists if and only if $\rho \in[0, \rho(\Delta)]$. If $\Delta>2$ firm 1 's expected profits when $k_{2}=\frac{\theta^{H}}{2}$ and $k_{1} \leq k_{2}$ are given by

$\pi_{1}\left(k_{1}, \frac{\theta^{H}}{2}\right)=\left\{\begin{array}{ccc}\rho \frac{k_{1}}{\theta^{L}}\left(\theta^{L}-k_{1}\right)+(1-\rho) k_{1}-c k_{1} & \text { if } & 0 \leq k_{1}<\theta^{L} \\ 0+(1-\rho) k_{1}-c k_{1} & \text { if } & \theta^{L} \leq k_{1} \leq \frac{\theta^{H}}{2}\end{array}\right.$

Since the profit function is concave in $k_{1}$ up to $k_{1}<\theta^{L}$ and increasing thereafter, it has two local maxima: one at $k_{1}^{*}=\min \left(\frac{1 c}{2 \rho} \theta^{L}, \theta_{L}\right)$ and the other one at $\frac{\theta^{H}}{2}$. As $k_{1}^{*}$ is decreasing in $\rho$ with $k_{1}^{*}=\theta^{L}$ at $\rho=\frac{1 c}{2}$, it trivially follows that the global maximum is $\frac{\theta^{H}}{2}$ for all $\rho \leq \frac{1 c}{2}$. In contrast, if $\rho \in\left(\frac{1 c}{2}, 1-c\right)$, we have

$\pi_{1}\left(\frac{\theta^{H}}{2}, \frac{\theta^{H}}{2}\right)-\pi_{1}\left(\frac{1-c}{2 \rho} \theta^{L}, \frac{\theta^{H}}{2}\right) \geq 0$ if and only if $\rho \leq \hat{\rho}(\Delta)$,

where $\hat{\rho}(\Delta)=\frac{1 c}{2}\left[1+\sqrt{\frac{\Delta}{\Delta}}\right] \in\left(\frac{1 c}{2}, 1-c\right)$ with $\lim _{\Delta \rightarrow 2} \hat{\rho}(\Delta)=$ $\frac{1 c}{2}$ Hence, $\left(\frac{\theta^{H}}{2}, \frac{\theta^{H}}{2}\right)$ is a symmetric equilibrium if $\rho \in[0, \hat{\rho}(\Delta)]$.

Assume now that $\Delta \leq 2$ so that firm 1 's expected profits when $k_{2}=\frac{\theta^{H}}{2}$ and $k_{1} \leq k_{2}$ are given by,

$\pi_{1}\left(k_{1}, \frac{\theta^{H}}{2}\right)=\left\{\begin{array}{rrrrr}(1 & c) k_{1} & & \text { if } \quad 0 \leq k_{1} \leq \theta^{L} & \frac{\theta^{H}}{2} \\ \rho \frac{k_{1}}{\theta^{H} / 2}\left(\begin{array}{ll}\theta^{L} & k_{1}\end{array}\right)+\left(\begin{array}{lllll}1 & \rho\end{array}\right) k_{1} & c k_{1} & \text { if } & \theta^{L} & \frac{\theta^{H}}{2} \leq k_{1} \leq \frac{\theta^{H}}{2}\end{array}\right.$

which has a local maximum at $k_{1}^{\prime}=\min \left(\frac{\theta^{L}}{2}+\frac{\theta^{H}}{4 \rho}(1-c-\rho), \frac{\theta^{H}}{2}\right)$. Note that $k_{1}^{\prime}$ is decreasing in $\rho$ and it is equal to $\frac{\theta^{H}}{2}$ for any $\rho \leq \tilde{\rho}(\Delta)=$ $\frac{1 c}{2}\left(\frac{2 \Delta}{3 \Delta 2}\right)$. In contrast for $\rho \in(\tilde{\rho}, 1-c), k_{1}^{\prime}=\frac{\theta^{L}}{2}+\frac{\theta^{H}}{4 \rho}(1-c-\rho)<\frac{\theta^{H}}{2}$ so that a symmetric equilibrium fails to exist.

We next show that there is a continuum of asymmetric equilibrium for any value of $\rho$ and $\Delta$. If $\Delta>2$ whenever firm 2 expands its capacity beyond $\frac{\theta^{H}}{2}$ firm 1 's expected profits for capacities $k_{1} \in\left[\theta^{H}-k_{2}, k_{2}\right]$ are given by

$\rho \min \left\{\frac{k_{1}}{\theta^{L}}\left[\theta^{L}-k_{1}\right], 0\right\}+(1-\rho) \frac{k_{1}}{k_{2}}\left(\theta^{H}-k_{1}\right)-c k_{1}$,

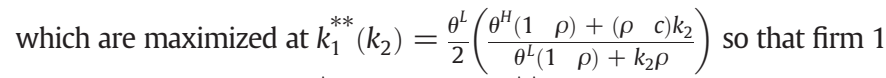
optimal choice is either $k_{1}^{*}$ or $\theta^{H}-k_{2}$ or $k_{1}^{* *}\left(k_{2}\right)$.

Assume first that $\rho \leq \frac{1}{2}$. As $1-\rho-c>0$, firm 1 is better off investing $\theta^{\mathrm{H}}-k_{2}$ rather than any smaller quantity. Moreover, $k_{1}^{* *}\left(k_{2}\right) \leq \theta^{H}-k_{2}$ for all $k_{2}<y(\rho)$, the capacity $\theta^{H}-k_{2}$ is firm 1 's best reply to any $k_{2} \in\left(\frac{\theta^{H}}{2}, y(\rho)\right]$, where



Consequently if $\rho \leq \frac{1 c}{2}$ there exists a continuum of asymmetric equilibria made of pairs $\left(\theta^{H}-k^{+}, k^{+}\right)$with $k^{+} \in\left(\frac{\theta^{H}}{2}, y(\rho)\right]$. Note that as $\rho \rightarrow 0$ the set of equilibria converges to the set of equilibria when demand is $\theta^{H}$ with probability one, i.e., $k^{+} \in\left[\frac{\theta^{H}}{2}, \frac{\theta^{H}}{2 c}\right]$ and $k^{-}=\theta^{H}-k^{+}$.

Assume now that $\rho \in\left(\frac{1 c}{2}, \hat{\rho}\right]$, so that producing below $\theta^{L}$, in particular at $k_{1}^{*}$, may turn an optimal choice. For these values of $\rho$ there are two disjoint sets of equilibria: one made of pairs $\left(\theta^{H}-k^{+}, k^{+}\right)$ with $k^{+} \in\left[\frac{\theta^{H}}{2}, \theta^{H}-\frac{\left(\begin{array}{lll}1 & c\end{array}\right)^{2}}{4 \rho\left(\begin{array}{lll}1 & \rho & c\end{array}\right)} \theta^{L}\right]$, and the other one consisting of pairs $\left(\theta^{H}-k^{+}, k^{+}\right)$with $k^{+} \in\left[\theta^{H}-\frac{1 c}{2 \rho} \theta^{L}, y(\rho)\right]$, where $\theta^{H}-\frac{\left(\begin{array}{ll}1 & c\end{array}\right)^{2}}{4 \rho(1 \quad \rho c)} \theta^{L} \leq$ $\theta^{H}-\frac{1 c}{2 \rho} \theta^{L} \leq y(\rho)$. Note that as $\rho \rightarrow \frac{1 c}{2}$ we have

$\lim _{\rho \rightarrow \frac{1}{2} c}\left[\theta^{H}-\left(\theta^{H}-\frac{\theta^{L}(1-c)^{2}}{4 \rho(1-\rho-c)}\right)-\frac{1-c}{2 \rho} \theta^{L}\right]=0$,

so that the two sets of equilibria become connected and hence we get the same equilibria as when $\rho \leq \frac{1 c}{2}$.

Finally, if $\rho \in(\hat{\rho}, 1-c)$, the only equilibrium set is made of the capacity pairs $\left(\theta^{H}-k^{+}, k^{+}\right)$with $k^{+} \in\left[\theta^{H}-\frac{(1 c)}{2 \rho} \theta^{L}, y(\rho)\right]$.

Assume now that $\Delta \leq 2$. When this is the case there are asymmetric equilibria that depend on the value of $\Delta$. For instance, if $\Delta>3 / 2$ the set of asymmetric equilibria is given by $k^{+} \in\left[\rho \frac{2 \theta^{H} \theta^{L}}{1 c+\rho}, y(\rho)\right]$ if $\rho \in\left(\tilde{\rho}, \frac{1 c}{2} \frac{1}{\Delta 1}\right)$, and by $k^{+} \in\left[\theta^{H}-\frac{1 c}{2 \rho} \theta^{L}, y(\rho)\right]$ if $\rho \in\left(\frac{1 c}{2} \frac{1}{\Delta 1}, 1-c\right)$. The complete characterization of the set of asymmetric equilibria for this last case is a tedious and mechanical exercise and it is hence omitted. ${ }^{20}$ Q.E.D.

Proof of Proposition 7. We prove part (ii) only as part (i) follows directly from Proposition 1. Standard arguments show that a pure strategy equilibrium does not exist. To see this, note first that in a pure strategy equilibrium both firms' prices must be equal. Otherwise, the firm with the low price could profitably increase its price towards its rival's without losing output. Next, this common price cannot exceed (zero) marginal costs; if it did, some firm could profitably deviate to a slightly lower price, thereby increasing its expected output with only a

\footnotetext{
${ }^{20}$ The interested reader can find the complete characterization and all the details in the working paper version of this paper.
} 
negligible effect on the expected price. Lastly, pricing at marginal costs cannot constitute an equilibrium either, since at least the large firm could obtain positive expected profits in the event that demand exceeds its rival's capacity if it raised its own price.

Accordingly, we next characterize the unique mixed strategy equilibrium. To do so, we concentrate our attention on the case in which $K \leq 1$, as $K>1$ will never be part of a subgame perfect equilibrium (the large firm would never sell at capacity and could increase its expected profits by reducing its capacity to $1-k^{-}$).

Let $F_{i}(p)=\operatorname{Pr}\left\{p_{i} \leq p\right\}$ denote the equilibrium mixed strategy of firm $i, i=1,2$, with $f_{i}(p)=F_{i}^{\prime}(p)$, and let $S_{i}$ be the support of $F_{i}$. Standard arguments imply that $S_{1} \cap S_{2}=[p, 1)$ and that $F_{1}$ and $F_{2}$ do not have mass points on $(p, 1) .^{21}$

Firm $i$ 's expected profits when pricing at $p$ are given by

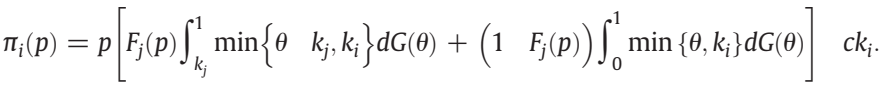

A necessary condition for firm $i$ to follow a mixed strategy is that $\pi_{i}(p)=\bar{\pi}_{i}$ for all $p \in S_{i}$, implying

$F_{j}(p)=\frac{\frac{\bar{\pi}_{i}+c k_{i}}{p}-\int_{0}^{1} \min \left\{\theta, k_{i}\right\} d G(\theta)}{\int_{k_{j}}^{1} \min \left\{\theta-k_{j}, k_{i}\right\} d G(\theta)-\int_{0}^{1} \min \left\{\theta, k_{i}\right\} d G(\theta)}$.

Using the boundary condition $F_{j}(p)=0$, it follows that

$\bar{\pi}_{i}+c k_{i}=p \int_{0}^{1} \min \left\{\theta, k_{i}\right\} d G(\theta)$.

Hence, for prices in the support $S_{i}$, it holds that

$F_{j}(p)=\frac{\int_{0}^{1} \min \left\{\theta, k_{i}\right\} d G(\theta)}{\int_{0}^{1} \min \left\{\theta, k_{i}\right\} d G(\theta)-\int_{k_{j}}^{1} \min \left\{\theta-k_{j}, k_{i}\right\} d G(\theta)} \frac{p-p}{p}$.

Let $F^{-}$and $F^{+}$denote the equilibrium mixed strategy of the small and the large firm, respectively. We first show that $F^{-}(p) \geq F^{+}(p)$. It follows that we cannot have $\lim _{p \uparrow 1} F^{+}(p)=1$, since this would imply $\lim _{p \uparrow 1} F^{-}(p) \geq 1$, with strict inequality for $k^{-}<k^{+}$. Consequently, we have the boundary condition $\lim _{p \uparrow 1} F^{-}(p)=1$, from which it follows that

$$
p=\frac{\int_{k}^{1} \min \left\{\theta-k, k^{+}\right\} d G(\theta)}{\int_{0}^{1} \min \left\{\theta, k^{+}\right\} d G(\theta)}<1 .
$$

Equilibrium expected profits become

$\pi_{i}=\frac{\int_{0}^{1} \min \{\theta, k\} d G(\theta)}{\int_{0}^{1} \min \left\{\theta, k^{+}\right\} d G(\theta)} \int_{k}^{1} \min \left\{\theta-k, k^{+}\right\} d G(\theta)-c k$, and

$\pi_{i}^{+}=\int_{k}^{1} \min \left\{\theta-k, k^{+}\right\} d G(\theta)-c k^{+}$.

Q.E.D.

Proof of Proposition 9. The proof uses the same arguments displayed in the Proof of Proposition 4. Recall that equilibrium existence can be guaranteed if the three following conditions hold:

\footnotetext{
21 If there were a mass point in the interior of the distribution, it would be optimal for the rival to transfer some probability weight below the mass point so as to increase the probability of having the low price. This is not feasible at the upper bound $(p=1)$ as the rival is pricing below this price with probability one. Hence, there can be mass points in the mixed strategies at the upper bound.
}

(i) $\pi^{-}$and $\pi^{+}$are submodular; (ii) $\left.\lim _{k_{i} \downarrow k} \frac{\partial \pi_{i}^{+}}{\partial k_{i}}\right|_{\left(k_{i}, k\right)}-\left.\lim _{k_{i} \uparrow k} \frac{\partial \pi_{i}}{\partial k_{i}}\right|_{\left(k_{i}, k\right)}>0$; and (iii) Both $\left.\frac{\partial \pi_{i}^{+}}{k_{i}}\right|_{(1,1)}<0$ and $\left.\frac{\partial \pi_{i}}{\partial k_{i}}\right|_{(0,0)}>0$ hold, so that $(0,0)$ and $(1,1)$ can be ruled out as equilibrium outcomes. Condition (ii) has already been shown to hold. Condition (iii) holds as $\left.\frac{\partial \pi_{i}^{+}}{\partial k_{i}}\right|_{(1,1)}=-c<0$ and $\left.\frac{\partial \pi_{i}}{\partial k_{i}}\right|_{(0,0)}=1-c>0$, where the last result follows from the fact that $\left.\frac{\partial \pi_{i}}{\partial k_{i}}\right|_{(0,0)}=\lim _{k \uparrow 0}\left(\left.\frac{\partial \pi_{i}}{\partial k_{i}}\right|_{(k, k)}\right)-c$, which equals $1-c$ from l'Hôpital's rule. Consequently, we only need to show that both cross partial derivatives are negative, i.e., $\frac{\partial^{2} \pi_{i}^{+}}{\partial k^{+} \partial k}<0$ and $\frac{\partial^{2} \pi_{i}}{\partial k \partial k^{+}}<0$, as this will ensure that condition (i) holds too.

For the large firm the result follows trivially as

$$
\frac{\partial^{2} \pi_{i}^{+}}{\partial k^{+} \partial k}=-g(K)<0
$$

For the small firm, $\frac{\partial^{2} \pi_{i}}{\partial k \partial k^{+}}=\frac{N\left(k, k^{+}\right)}{\left(h\left(k^{+}\right)\right)^{2}}$, where the numerator $N\left(k^{-}, k^{+}\right)$ is given by

$$
\begin{aligned}
& {\left[\left(1 \quad G\left(k^{-}\right)\right)(1 \quad G(K)) \quad g(K) \int_{0}^{1} \min \left\{\theta, k^{-}\right\} d G(\theta)\right] \int_{0}^{1} \min \left\{\theta, k^{+}\right\} d G(\theta)}
\end{aligned}
$$

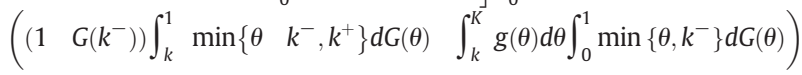

$$
\begin{aligned}
& \times\left(1 \quad G\left(k^{+}\right)\right) .
\end{aligned}
$$

Since

$$
\begin{aligned}
\int_{z}^{1} \min \{\theta-z, y\} d G(\theta) & =\int_{z}^{z+y}(\theta-z) d G(\theta)+y(1-G(x+y)) \\
& =\int_{0}^{y} \theta d G(\theta+z)+y(1-G(x+y))
\end{aligned}
$$

straightforward computations allow us to rewrite $N\left(k^{-}, k^{+}\right)$as follows

$$
\begin{aligned}
& \left(1 \quad G\left(k^{-}\right)\right)\left(\left[\begin{array}{ll}
1 & G(K)
\end{array}\right] \int_{0}^{k^{+}} \theta d G(\theta) \quad\left(1 \quad G\left(k^{+}\right)\right) \int_{0}^{k^{+}} \theta d G\left(\theta+k^{-}\right)\right) \\
& \left(\int_{0}^{1} \min \left(\theta, k^{-}\right) d G(\theta)\right)\left(g(K) \int_{0}^{1} \min \left(\theta, k^{+}\right) d G(\theta) \quad\left(1 \quad G\left(k^{+}\right)\right) \int_{k}^{K} g(\theta) d \theta\right) .
\end{aligned}
$$

If $g$ is non decreasing (i.e., under Assumption 5) $N\left(k^{-}, k^{+}\right)$is negative. Note that for a non decreasing $g$ it holds that $\int_{0}^{k^{+}} \theta d G(\theta+k) \geq\left(\int_{0}^{k^{+}} \theta d G(\theta)\right)$, which combined with $1-G\left(k^{+}\right) \geq$ $1-G(K)$ ensures that the first term is negative. Similarly, the second one is negative as $g(K) \int_{0}^{1} \min \left(\theta, k^{+}\right) d G(\theta) \geq g(K) k^{+}\left(1-G\left(k^{+}\right)\right)$, and $g(K) k^{+} \geq\left(\int_{k}^{K} g(\theta) d \theta\right)$. It hence follows that $N\left(k^{-}, k^{+}\right)<0$, so that $\frac{\partial^{2} \pi_{i}}{\partial k \partial k^{+}}=\frac{N\left(k, k^{+}\right)}{\left(h\left(k^{+}\right)\right)^{2}}<0$, as claimed.

Regarding uniqueness, we next show for all $\left(\tilde{k}, \tilde{k}^{+}\right)$such that $\left.\frac{\partial \pi_{i}}{\partial k_{i}}\right|_{\left(\tilde{k}, \tilde{k}^{+}\right)}=\left.\frac{\partial \pi_{i}^{+}}{\partial k_{i}}\right|_{\left(\tilde{k}, \tilde{k}^{+}\right)}=0$ it is satisfied that $\left|\frac{\partial^{2} \pi_{i}}{\partial k \partial k^{+}}\right|_{\left(\tilde{k}, \tilde{k}^{+}\right)} \mid-$ $\left|\frac{\partial^{2} \pi_{i}^{+}}{\partial k^{+} \partial k}\right|_{\left(\tilde{k}, \tilde{k}^{+}\right)}<0$.

Let $\left(\tilde{k}, \tilde{k}^{+}\right)$be such that $\left.\frac{\partial \pi_{i}}{\partial k_{i}}\right|_{\left(\tilde{k}, \tilde{k}^{+}\right)}=\left.\frac{\partial \pi_{i}^{+}}{\partial k_{i}}\right|_{\left(\tilde{k}, \tilde{k}^{+}\right)}=0$ holds. Denote by $J\left(\tilde{k}, \tilde{k}^{+}\right)$the second order cross derivative of the small firm evaluated at $\left(\tilde{k}, \tilde{k}^{+}\right)$, i.e., let $J\left(\tilde{k}, \tilde{k}^{+}\right)=\frac{\partial^{2} \pi_{i}}{\partial k \partial k^{+}} \mid\left(\tilde{k}, \tilde{k}^{+}\right)$. Straightforward computations give

$$
\begin{aligned}
J\left(\tilde{k}, \tilde{k}^{+}\right) & =\frac{(1-G(\tilde{k})) c-g(\tilde{K}) \int_{0}^{1} \min \{\theta, \tilde{k}\} d G(\theta)-c\left(1-G\left(\tilde{k}^{+}\right)\right)}{\int_{0}^{1} \min \left\{\theta, \tilde{k}^{+}\right\} d G(\theta)} \\
& =\frac{\left(G\left(\tilde{k}^{+}\right)-G(\tilde{k})\right) c-g(\tilde{K}) \int_{0}^{1} \min \{\theta, \tilde{k}\} d G(\theta)}{\int_{0}^{1} \min \left\{\theta, \tilde{k}^{+}\right\} d G(\theta)} .
\end{aligned}
$$


Since for any non decreasing $g$, it holds true that $J\left(\tilde{k}, \tilde{k}^{+}\right)<0$, the sign of $\left.\left|\frac{\partial^{2} \pi_{i}}{\partial k \partial k^{+}}\right|_{\left(\tilde{k}, \tilde{k}^{+}\right)}|-| \frac{\partial^{2} \pi_{i}^{+}}{\partial k^{+} \partial k}\right|_{\left(\tilde{k}, \tilde{k}^{+}\right)} \mid$equals the sign of

$$
\left(G\left(\tilde{k}^{+}\right) G\left(\tilde{k}^{-}\right)\right) c+g(\tilde{K})\left(\int_{0}^{1} \min \left\{\theta, \tilde{k}^{-}\right\} d G(\theta) \quad \int_{0}^{1} \min \left\{\theta, \tilde{k}^{+}\right\} d G(\theta)\right)<0,
$$

which ensures uniqueness, as claimed. Q.E.D.

Proof of Lemma 1. Assume that demand is uniformly distributed in the unit interval. Suppose first that firms face demand uncertainty at the investment stage only. As a particular case of Proposition 5, equilibrium capacities $k^{-}$and $k^{+}$satisfy $k^{+}+k^{-}=1-c$ and $k^{+}=\alpha k^{-}$with $\alpha>2$ given implicitly by the equation $\alpha^{2}-2 \alpha \ln (\alpha)=\frac{3}{2}$. Total expected profits are given by,

$$
\Pi=(1-c)\left(k^{-}+k^{+}\right)-\left(k^{-}\right)^{2}\left(1+\frac{1}{2} \frac{k^{-}}{k^{+}}-\ln \left(\frac{k^{-}}{k^{+}}\right)\right)-\left(\frac{1}{2}\left(k^{+}\right)^{2}+k^{-} k^{+}\right) .
$$

At equilibrium capacities, these are approximately equal to $0.36(1-c)^{2}$.

Suppose now that firms face demand uncertainty at both the investment and pricing stages. Closed form solutions when demand is uniformly distributed are given by,

$k=\frac{1}{2}\left(2+c-\sqrt{ } 2+4 c+3 c^{2}\right)$,

$k^{+}=\frac{1}{2}\left(-3 c+\sqrt{ } 2+4 c+3 c^{2}\right)$.

Total expected profits are given by,

$\Pi=\pi_{i}+\pi_{j}^{+}=\frac{1}{2}\left(2-2 k-k^{+}\right) \frac{(2-k) k+\left(2-k^{+}\right) k^{+}}{2-k^{+}}-c\left(k+k^{+}\right)$

Substituting for $k^{-}$and $k^{+}$from Eqs. (13) and (14) above, we find equilibrium expected profits in reduced form,

$$
\begin{aligned}
\Pi= & \frac{1}{4}\left(c+\sqrt{ } 2+4 c+3 c^{2}\right) \frac{(1+2 c) \sqrt{ } 2+4 c+3 c^{2}-c(5+4 c)}{2+\frac{1}{2}\left(3 c-\sqrt{ } 2+4 c+3 c^{2}\right)} \\
& -c(1-c) .
\end{aligned}
$$

It is straightforward to check that Eq. (15) exceeds $0.36(1-c)^{2}$. Hence, expected profits are always lower when demand is uncertain at the investment stage only. Since investment and output are equal under both games, prices must also be lower. Q.E.D.

\section{Appendix A.3. Comparison with other games}

Proof of Lemma 2. (i) Subgame perfect equilibrium aggregate capacity is given by $K^{u}=G^{1}(1-c)$ in the game with continuous demand uncertainty, and it is given by $K^{c}=E[\theta]$ in the certainty equivalent game. The difference $K^{u}-K^{c}$ is strictly decreasing in $c$. Furthermore, $\lim _{c \rightarrow 0}\left(K^{u}-K^{c}\right)>0$ and $\lim _{c \rightarrow 1}\left(K^{u}-K^{c}\right)<0$. Hence, it follows that there must exist some $\widehat{c}$ such that $K^{u}>K^{c}$ if and only if $c \in(0, \widehat{c})$.

(ii) Let $W^{u}$ and $W^{c}$ denote subgame perfect equilibrium welfare in the game with demand uncertainty and in the certainty equivalent game, respectively. These can be expressed as,

$$
\begin{aligned}
W^{u} & =\int_{0}^{1} \min \left\{\theta, K^{u}\right\} d G(\theta)-c K^{u}, \\
W^{c} & =\int_{0}^{1} \theta d G(\theta)-c \int_{0}^{1} \theta d G(\theta) .
\end{aligned}
$$

Since $K^{u}<1$ total surplus is smaller in the uncertain game as for a positive mass of demand realizations total surplus is not maximal. However, since the ranking of total production costs across games depends on $c$ as shown in (i), the sign of $W^{u}-W^{c}$ is a priori unclear. Computing the difference in total welfare in the two games we have that

$$
\left.W^{u}-W^{c}=c\left(E[\theta]-G^{1}(1-c)\right)-\int_{G^{1}(1}^{1} c\right)\left(\theta-G^{1}(1-c)\right) d G(\theta) .
$$

The above expression is clearly negative if $c \in(0, \widehat{c})$, as the second term is negative and, by point (i) above, the first term is negative as well. Furthermore, since $\frac{\partial\left(W^{u} W^{c}\right)}{\partial c}=E[\theta]-G^{1}(1-c)>0$ for $c \in(\widehat{c}, 1)$ and $\lim _{c \uparrow 1}\left(W^{u}-W^{c}\right)=0$, it follows that $W^{u}<W^{c}$ for all $c$.

Finally note that $E[\theta]$ maximizes welfare at the certainty equivalent game. Similarly, $G^{1}(1-c)$ maximizes $W^{u}$, as $\frac{\partial W^{u}}{\partial K}=1-G(K)-c=0$ implies $K^{F B}=G^{1}(1-c)$.

(iii) and (iv). In the certainty equivalent game, subgame perfect equilibrium prices are equal to consumers' reservation price, so that consumer surplus is zero. In the game with continuous demand uncertainty, prices are strictly below the reservation price for $\theta \in\left(0, G^{1}(1-c)\right) \subset[0,1]$, so that consumer surplus is strictly positive. It follows that expected prices must be lower and consumer surplus higher under demand uncertainty. Finally, for $\theta \in(k, K)$, the equilibrium is in mixed strategies, so there is price dispersion. Q.E.D.

Proof of Lemma 3. The first order, second order and cross derivatives of firms' profits functions in the Cournot game are given by

$$
\begin{aligned}
& \frac{\partial \pi_{i}}{\partial q_{i}}=1-G\left(q_{i}+q_{j}\right)-c=0 \text { and } \\
& \frac{\partial^{2} \pi_{i}}{\partial q_{i}^{2}}=\frac{\partial^{2} \pi_{i}}{\partial q_{i} \partial q_{j}}=-g\left(q_{i}+q_{j}\right)<0 .
\end{aligned}
$$

By solving the FOC, any output pair $\left(q_{1}, q_{2}\right)$ that satisfies $q_{1}+q_{2}=G^{1}(1-c)$ constitutes an equilibrium of the Cournot game with uncertain and inelastic demand. Q.E.D.

\section{References}

Acemoglu, D., Bimpikis, K., Ozdaglar, A., 2009. Price and capacity competition. Games and Economic Behavior 66, 1-26.

Amir, R., Wooders, J., 2000. One-way spillovers, endogenous innovator/imitator roles and research joint ventures. Games and Economic Behavior 31, 1-25.

Amir, R., García, F., Knauff, M., 2010. Symmetry-breaking in two-player games via strategic substitutes and diagonal nonconcavity: a synthesis. Journal of Economic Theory 145, 1968-1986.

Barlow, R.E., Proschan, F., 1965. Mathematical Theory of Reliability. John Wiley and Sons, New York.

Cripps, M. W. and N. J. Ireland (1988) "Equilibrium and Capacities in a Market of Fixed Size", manuscript.

Davidson, C., Deneckere, R., 1986. Long-run competition in capacity, short-run competition in price and the Cournot model. The Rand Journal of Economics 17, 404-415.

De Frutos, M.-A., Fabra, N., 2007. Endogenous Capacities and Price Competition: the Role of Demand Uncertainty. CEPR Discussion Paper 6096

Fabra, N., von der Fehr, N.-H., de Frutos, M.-A., 2009. Market Design and Investment Incentives. CEPR Discussion Paper 6626.

Gabszewicz, J., Poddar, S., 1997. Demand fluctuations and capacity utilization under duopoly. Economic Theory 10, 131-146.

Gal-Or, E., 1984. Price dispersion with uncertain demand. International Economic Review 25, 441-457.

Grimm, V., Zoettl, G., 2006. Capacity Choice under Uncertainty: the Impact of Market Structure. Working Paper Series, 23. University of Cologne.

Hviid, M., 1990. Sequential capacity and price choices in a duopoly model with demand uncertainty. Journal of Economics 51 (2), 121-144.

Klemperer, P., Meyer, M., 1989. Supply function equilibria in oligopoly under uncertainty. Econometrica 57, 1243-1277.

Kreps, D., Scheinkman, J., 1983. Quantity precommitment and Bertrand competition yield Cournot outcomes. Bell Journal of Economics 14, 326-337. 
Lepore, J. J. (2008) "Cournot Outcomes under Bertrand-Edgeworth Competition with Demand Uncertainty", mimeo, Orfalea Business College.

Osborne, M.J., Pitchik, C., 1986. Price competition in a capacity constrained duopoly. Journal of Economic Theory 38, 238-260.

Reynolds, S., Wilson, B., 2000. Bertrand-Edgeworth competition, demand uncertainty, and asymmetric outcomes. Journal of Economic Theory 92, 122-141.
Topkis, D., 1979. Equilibrium points in nonzero sum N-person submodular games. SIAM Journal on Control and Optimization 17, 773-787.

Vives, X., 1999. Oligopoly Pricing: Old Ideas and New Tools. MIT Press, Cambridge and London. 